(1)

CrossMark

\title{
Association between adiposity measures and COPD risk in Chinese adults
}

\author{
Jiachen $\mathrm{Li}^{1}$, Lu Zhu ${ }^{1}$, Yuxia Wei ${ }^{1}$, Jun Lv ${ }^{1,2,3}$, Yu Guo ${ }^{4}$, Zheng Bian ${ }^{4}$, \\ Huaidong Du ${ }^{5,6}$, Ling Yang ${ }^{5,6}$, Yiping Chen ${ }^{5,6}$, Yonglin Zhou ${ }^{7}$, Ruqin Gao ${ }^{8}$, \\ Junshi Chen ${ }^{9}$, Zhengming Chen ${ }^{6}$, Weihua Cao ${ }^{1}$, Canqing Yu $\mathbb{1}^{1}$ and Liming $\mathrm{Li}^{1}$ \\ on behalf of the China Kadoorie Biobank Collaborative Group ${ }^{10}$
}

Affiliations: ${ }^{1}$ Dept of Epidemiology and Biostatistics, School of Public Health, Peking University Health Science Center, Beijing, China. ${ }^{2}$ Key Laboratory of Molecular Cardiovascular Sciences (Peking University), Ministry of Education, Beijing, China. ${ }^{3}$ Peking University Institute of Environmental Medicine, Beijing, China. ${ }^{4}$ Chinese Academy of Medical Sciences, Beijing, China. ${ }^{5}$ Medical Research Council Population Health Research Unit at the University of Oxford, Oxford, UK. ${ }^{6}$ Clinical Trial Service Unit and Epidemiological Studies Unit (CTSU), Nuffield Dept of Population Health, University of Oxford, Oxford, UK. ${ }^{7}$ Jiangsu CDC, Nanjing, China. ${ }^{8}$ Qingdao CDC, Qingdao, China. ${ }^{9}$ China National Center for Food Safety Risk Assessment, Beijing, China.

${ }^{10}$ The members of the steering committee and collaborative group are listed in the supplementary material.

Correspondence: Canqing Yu, Dept of Epidemiology and Biostatistics, Peking University Health Science Center, 38 Xueyuan Road, Beijing 100191, China. E-mail: yucanqinglapku.edu.cn

@ERSpublications

Abdominal adiposity and underweight were risk factors for COPD in Chinese adults. Both BMI and measures of abdominal adiposity should be considered in the prevention of COPD. http://bit.ly/ $36 \mathrm{To} 4 \mathrm{fk}$

Cite this article as: $\mathrm{Li}$ J, Zhu L, Wei Y, et al. Association between adiposity measures and COPD risk in Chinese adults. Eur Respir J 2020; 55: 1901899 [https://doi.org/10.1183/13993003.01899-2019].

ABSTRACT Bodyweight and fat distribution may be related to COPD risk. Limited prospective evidence linked COPD to abdominal adiposity. We investigated the association of body mass index (BMI) and measures of abdominal adiposity with COPD risk in a prospective cohort study.

The China Kadoorie Biobank recruited participants aged 30-79 years from 10 areas across China. Anthropometric indexes were objectively measured at the baseline survey during 2004-2008. After exclusion of participants with prevalent COPD and major chronic diseases, 452259 participants were included and followed-up until the end of 2016. We used Cox models to estimate adjusted hazard ratios relating adiposity to risk of COPD hospitalisation or death.

Over an average of 10.1 years of follow-up, 10739 COPD hospitalisation events and deaths were reported. Compared with subjects with normal BMI $\left(18.5-<24.0 \mathrm{~kg} \cdot \mathrm{m}^{-2}\right)$, underweight $\left(\mathrm{BMI}<18.5 \mathrm{~kg} \cdot \mathrm{m}^{-2}\right.$ ) individuals had increased risk of COPD, with adjusted hazard ratio 1.78 (95\% CI 1.66-1.89). Overweight (BMI $24.0-<28.0 \mathrm{~kg} \cdot \mathrm{m}^{-2}$ ) and obesity $\left(B M I \geqslant 28.0 \mathrm{~kg} \cdot \mathrm{m}^{-2}\right.$ ) were not associated with an increased risk after adjustment for waist circumference. A higher waist circumference $(\geqslant 85 \mathrm{~cm}$ for males and $\geqslant 80 \mathrm{~cm}$ for females) was positively associated with COPD risk after adjustment for BMI. Additionally, waist-to-hip ratio and waist-to-height ratio were positively related to COPD risk.

Abdominal adiposity and underweight were risk factors for COPD in Chinese adults. Both BMI and measures of abdominal adiposity should be considered in the prevention of COPD.

This article has an editorial commentary: https://doi.org/13993003.00107-2020

This article has supplementary material available from erj.ersjournals.com

Received: 25 Sept 2019 | Accepted after revision: 20 Dec 2019

Copyright OERS 2020. This version is distributed under the terms of the Creative Commons Attribution NonCommercial Licence 4.0. 


\section{Introduction}

COPD is a global health problem, causing $\sim 3$ million deaths every year [1]. In China, the prevalence of COPD is estimated to be $8.6 \%$ in adults aged $>20$ years, equivalent to 99.9 million prevalent cases [2]. Such a heavy disease burden has been a great concern. Identifying and modifying risk factors is essential for controlling the epidemic of COPD.

Adiposity is an established risk factor for a range of chronic diseases. Maintaining a suitable body weight has been considered as a major part of healthy lifestyle [3], but the relationship between adiposity and COPD risk is not fully understood. Evidence from cross-sectional studies implies an inverse correlation between body mass index (BMI) and COPD prevalence $[2,4]$. However, the observed relationship may not be causal, as COPD patients often undergo weight loss and tend to be thinner than healthy people [5]. Prospective cohort studies could address reverse causality, while available prospective evidence is not sufficient. In addition to general adiposity measured using BMI, fat distribution may be an important factor in the development of COPD. Abdominal adipose tissue contributes to systemic inflammation, which is related to the pathogenesis of COPD. One large cohort study in the United States found that obesity, in particular abdominal adiposity measured by waist circumference was associated with an increased risk of COPD [6]. There are ethnic variations in body size and composition. Generally, Chinese people have lower BMI and a higher percentage of body fat than Caucasians [7]. Whether the association between adiposity and COPD holds in the Chinese population is unclear.

The objective of this study is to examine the association of general and abdominal adiposity measures with risk of COPD hospitalisation or death in a population-based prospective cohort, the China Kadoorie Biobank (CKB) study.

\section{Methods}

\section{Study population}

Details of the CKB study have been described previously [8, 9]. Adults aged 30-79 years were recruited from 10 regions across China, including five urban and five rural regions (supplementary figure $\mathrm{S} 1$ ). The $\mathrm{CKB}$ study is not designed to be representative of Chinese population, and the cohort was not a random sample. However, the study selected 10 geographically diverse regions to represent major regional differences in disease patterns, lifestyles, and economic levels throughout China. In each administrative unit, all males and females who were permanently resident and without a major disability were identified and invited to participate. The participation rate was $\sim 30 \%$. A total of 512715 participants completed the baseline survey. The sample size of 0.5 million was determined to achieve acceptable statistical power to detect the complex interplay between environmental and genetic factors of common chronic diseases. The $\mathrm{CKB}$ study was approved by the ethical committee of the Chinese Center for Disease Control and Prevention (Beijing, China) and the Oxford Tropical Research Ethics Committee, University of Oxford (Oxford, UK). Signed informed consent was obtained from all participants.

\section{Assessment of exposure and covariates}

Anthropometric measurements were collected by trained staff according to standard procedures [10] at the baseline survey between June 2004 and July 2008. Standing height was measured without shoes, to the nearest $0.1 \mathrm{~cm}$, using a stadiometer. Weight was measured without shoes but in light clothing, to the nearest $0.1 \mathrm{~kg}$, using the TBF-300GS Body Composition Analyser (Tanita Inc., Tokyo, Japan). The weight of clothing was estimated and subtracted according to the season. Waist and hip circumference were measured to the nearest $0.1 \mathrm{~cm}$ using soft tape. The reliability of anthropometric measurements was evaluated in a re-survey among a randomly chosen $5 \%$ of participants in 2008 . The correlation coefficients between baseline and re-survey measures were quite high for height (0.99), weight (0.96), waist circumference (0.84) and hip circumference (0.82). BMI was calculated as weight $(\mathrm{kg})$ divided by the square of height $\left(\mathrm{m}^{2}\right)$. Waist-to-hip ratio and waist-to-height ratio were calculated as waist circumference divided by hip circumference and height, respectively.

At the baseline survey, information on covariates was collected using an interviewer-administered questionnaire, including sociodemographic factors (age, sex, marital status, highest education), lifestyle (smoking, passive smoking, alcohol drinking, physical activity, diet, household air pollution) and medical history (respiratory symptoms, asthma, tuberculosis). Passive smoking was evaluated by asking if participants had lived with smoker in the same house and the duration of exposure. Household air pollution was evaluated by solid fuel use for cooking and heating in the house. A food frequency questionnaire comprising 12 major food groups was used to assess diet. Daily physical activity level was assessed by calculating the metabolic equivalent task-hours (MET-h). Prebronchodilator forced expiratory volume in $1 \mathrm{~s}\left(\mathrm{FEV}_{1}\right)$ and forced vital capacity $(\mathrm{FVC})$ were measured by trained technicians following recommended procedures [11]. 


\section{Assessment of outcome}

Participants were followed-up until the date of COPD (International Classification of Diseases-10 J41-J44) incidence, death, loss to follow-up or December 31,2016, whichever came first. The vital status and causes of death were obtained through official residential records and death certificates. Information on COPD morbidity was also collected through electronic linkage with the national health insurance system, which was established recently in all study regions and contained detail hospitalisation information. Incident cases from health insurance database were hospitalisation events, without including diagnoses made in an outpatient setting. Participants may be hospitalised and receive a COPD diagnosis due to COPD exacerbation, severe respiratory symptoms or comorbidities. Linkage with the health insurance database had been achieved for $98 \%$ of cohort participants. Active follow-up was conducted annually for participants who failed to be linked to the local health insurance database. In active follow-up, the interviewer asked participants if they had received a diagnosis of COPD by a physician during the past year. $<1 \%$ (4875) of participants were lost to follow-up. To evaluate the validity of COPD diagnosis in the CKB study, 1069 randomly selected cases were adjudicated. A diagnosis of COPD was confirmed in $85 \%$ of reported cases [12].

\section{Statistical analyses}

We excluded participants who had been diagnosed with chronic bronchitis or emphysema by a physician ( $\mathrm{n}=13288$ ), or those who had airflow obstruction defined as $\mathrm{FEV}_{1} / \mathrm{FVC}$ ratio $<0.7(\mathrm{n}=27483$ ). In addition, we excluded participants with prevalent cancer $(n=2578)$, coronary heart disease $(n=15472)$, asthma $(\mathrm{n}=2806)$ or tuberculosis $(\mathrm{n}=7659)$, as these diseases may cause significant weight change. Two participants were additionally excluded because of missing BMI. Finally, 452259 participants were included in the present analysis.

General adiposity was defined based on BMI using cut-off points according to the guidelines for prevention and control of overweight and obesity in Chinese adults [13, 14]. Participants were categorised into four groups: underweight $\left(<18.5 \mathrm{~kg} \cdot \mathrm{m}^{-2}\right)$, normal $\left(18.5-<24.0 \mathrm{~kg} \cdot \mathrm{m}^{-2}\right)$, overweight $\left(24.0-<28.0 \mathrm{~kg} \cdot \mathrm{m}^{-2}\right)$ and obese $\left(\geqslant 28.0 \mathrm{~kg} \cdot \mathrm{m}^{-2}\right)$. Abdominal adiposity was evaluated by waist circumference, waist-to-hip ratio and waist-to-height ratio, according to previously recommended cut-off points $[13,15]$. Additionally, we used restricted cubic splines with four knots at the 5th, 35th, 65th and 95th percentiles of adiposity measures distribution to examine the nonlinear relationship.

Cox regression model was used to calculate the hazard ratios (HRs) and 95\% confidence intervals for the association between adiposity measures and risk of COPD hospitalisation or death, with age as the time scale, stratified jointly by regions and 5-year birth cohorts. Potential confounders were adjusted for in the multivariate Cox model: sex, education, marital status, smoking status, passive smoking, cooking and heating fuel type, alcohol drinking, physical activity (MET-h.day ${ }^{-1}$ ), intake frequencies of red meat, fresh fruit and vegetables, and respiratory symptoms. These covariates were selected based on prior knowledge, including sociodemographic variables, established risk factors for COPD and health-related lifestyles, which may influence body weight. We used Schoenfeld residuals to check the proportional hazards assumption for all models and found no violation.

We examined the association in various subgroups defined by baseline characteristics: sex (male, female), area (urban, rural), age ( $<50$ years, 50-59 years, $\geqslant 60$ years), education level (primary school or lower, middle/high school, college or higher), smoking status (nonsmoker, current smoker), menopause (no, yes) and physical activity (three groups defined by sex-specific tertiles). Joint analysis of general and abdominal adiposity measures was performed. We conducted the following sensitivity analyses to test the robustness of our results: excluding participants who developed COPD in the first 3 years of follow-up; restricting the analysis in never-smokers; using the lower limit of normal (LLN) definition to exclude participants with airflow obstruction at baseline; and excluding participants with diabetes at baseline. All analyses were performed using Stata (version 14.0; StataCorp, College Station, TX, USA). The significance level was set at 0.05.

\section{Results}

Among the 452259 participants, 182209 (40.3\%) were male and 255031 (56.4\%) were from rural areas. The mean \pm SD age at baseline was $51.1 \pm 10.4$ years. The overall mean \pm SD BMI was $23.7 \pm 3.3 \mathrm{~kg} \cdot \mathrm{m}^{-2}$, with $3.9 \%$ being underweight, $33.5 \%$ overweight and $10.5 \%$ obese. The overall mean \pm SD waist circumference was $80.3 \pm 9.6 \mathrm{~cm}$, with $41.9 \%$ being abdominally obese (waist circumference $\geqslant 85 \mathrm{~cm}$ for males; $\geqslant 80 \mathrm{~cm}$ for females). Participants with higher BMI were more likely to be urban residents, to consume fresh fruit, vegetables and red meat daily, and were less likely to be current smokers $(p<0.001)$. Compared with participants with normal BMI, those with lower or higher BMI were more likely to be older, female, physically inactive and have respiratory symptoms at baseline $(\mathrm{p}<0.001)$. Similar results were observed for abdominal adiposity categories defined by waist circumference, waist-to-hip ratio and waist-to-height ratio (table 1 and supplementary table $\mathrm{S} 1$ ). 
TABLE 1 Baseline characteristics of participants by baseline body mass index (BMI) and waist circumference $(n=452259)$

\begin{tabular}{|c|c|c|c|c|c|c|}
\hline & \multicolumn{4}{|c|}{ BMI $\mathrm{kg} \cdot \mathrm{m}^{-2}$} & \multicolumn{2}{|c|}{ Waist circumference $\mathrm{cm}$} \\
\hline & $<18.5$ & $18.5-<24.0$ & $24.0-<28.0$ & $\geqslant 28.0$ & $\begin{array}{c}<85 \text { (male) } \\
<80 \text { (female) }\end{array}$ & $\begin{array}{c}\geqslant 85 \text { (male) } \\
\geqslant 80 \text { (female) }\end{array}$ \\
\hline Participants n & 17579 & 235379 & 151649 & 47652 & 262902 & 189357 \\
\hline Age years mean & 53.8 & 50.7 & 51.3 & 51.9 & 49.3 & 52.8 \\
\hline Female & 61.0 & 58.1 & 60.0 & 66.1 & 57.1 & 63.3 \\
\hline Urban & 31.3 & 38.3 & 49.7 & 55.1 & 38.3 & 50.9 \\
\hline Married & 88.6 & 90.6 & 92.3 & 92.1 & 90.7 & 91.9 \\
\hline Primary school or lower & 51.5 & 49.4 & 49.1 & 51.9 & 49.5 & 49.8 \\
\hline Current smoker & 32.4 & 27.9 & 24.0 & 23.0 & 27.4 & 24.7 \\
\hline Passive smoker & 45.7 & 44.7 & 44.4 & 44.9 & 44.8 & 44.4 \\
\hline Cooking with solid fuel & 40.2 & 36.9 & 35.4 & 35.1 & 37.3 & 35.0 \\
\hline Heating with solid fuel & 38.5 & 37.3 & 36.9 & 37.4 & 37.3 & 37.1 \\
\hline Drinking weekly & 13.9 & 15.1 & 14.8 & 14.5 & 14.6 & 15.3 \\
\hline Consuming fruit daily & 14.9 & 18.2 & 19.0 & 19.3 & 18.2 & 19.0 \\
\hline Consuming fresh vegetables daily & 93.1 & 94.4 & 95.1 & 95.1 & 94.3 & 95.1 \\
\hline Consuming meat daily & 25.8 & 28.3 & 30.7 & 31.4 & 28.1 & 31.1 \\
\hline Physical activity MET-h.day ${ }^{-1}$ & 21.5 & 22.2 & 21.3 & 20.1 & 22.2 & 20.8 \\
\hline Respiratory symptoms ${ }^{\#}$ & 13.0 & 11.3 & 12.6 & 16.1 & 11.5 & 13.4 \\
\hline \multicolumn{7}{|c|}{$\begin{array}{l}\text { Data are presented as } \% \text {, unless otherwise stated. Linear (for continuous variable) or logistic (for } \\
\text { categorical variables) models were used to calculate predictive margins adjusting for age, sex and region. } \\
\text { \#: defined as the presence of any one of the following symptoms: 1) usually become short of breath when } \\
\text { walking on level ground; 2) usually slow down due to chest discomfort when walking on level ground; } \\
\text { 3) cough frequently during the past } 12 \text { months; and 4) cough up sputum after getting up in the morning } \\
\text { during the past } 12 \text { months. }\end{array}$} \\
\hline
\end{tabular}

\section{General adiposity and COPD}

During a median follow-up of 10.1 years (interquartile range 2.2-11.1 years), we documented 10739 COPD hospitalisation events and deaths. The overall incidence rate for hospitalisation or death from COPD was 2.40 per 1000 person-years. Individuals with a BMI of $<18.5 \mathrm{~kg} \cdot \mathrm{m}^{-2}$ had a higher incidence rate (7.08 per 1000 person-years). After adjusting for major covariates, underweight and obese participants had HRs (95\% CI) of 1.75 (1.64-1.86) and 1.10 (1.02-1.19), respectively, as compared with participants with normal BMI. The association between obesity and COPD disappeared after additional adjustment for waist circumference, while underweight remained a statistically significant risk factor, with HR (95\% CI) $1.78(1.66-1.89)$

We observed that the association between BMI and COPD differed by sex ( $\mathrm{p}$-value for interaction $<0.001$ ), and the stratified results are presented in table 2. BMI was inversely associated with COPD risk in males. Overweight and obesity appeared to be protective factors, with adjusted HRs (95\% CI) being $0.84(0.77-0.90)$ and $0.76(0.65-0.90)$. The inverse association was weaker in females than in males, and no statistically significant association was found between obesity and COPD in females. Additionally, we used restricted cubic splines to investigate the nonlinear relationship between BMI and COPD. In males, COPD risk decreased with higher BMI and levelled off at a BMI of $23 \mathrm{~kg} \cdot \mathrm{m}^{-2}$. In females, the lowest risk of COPD was at BMI $\sim 23-24 \mathrm{~kg} \cdot \mathrm{m}^{-2}$, and BMI $>24 \mathrm{~kg} \cdot \mathrm{m}^{-2}$ appeared to be associated with increased risk of COPD (figure 1). The association between BMI and COPD risk was modified by baseline age, area, smoking status and level of physical activity (supplementary table S2).

\section{Abdominal adiposity and COPD}

Overall, participants in the lowest waist circumference group had the highest crude incidence rate for hospitalisation or death from COPD (4.78 per 1000 person-years). After adjustment for covariates and BMI, abdominal adiposity was associated with COPD, and the risk increased with higher waist circumference. The adjusted HR $(95 \% \mathrm{CI})$ for the highest waist circumference group $(\geqslant 95 \mathrm{~cm}$ for males; $\geqslant 90 \mathrm{~cm}$ for females) was $1.57(1.43-1.73)$ compared with normal group (waist circumference $70-<85 \mathrm{~cm}$ for males; $65-<80 \mathrm{~cm}$ for females) (table 3 ). The positive trend for abdominal adiposity and COPD was similar in males and females, while the association between lower waist circumference and COPD was limited to females. Based on models with spline terms, waist circumference was positively associated with COPD in males, and those with waist circumference $<75 \mathrm{~cm}$ had the lowest risk. In females, waist 
TABLE 2 Association between baseline body mass index (BMI) and risk of COPD

\begin{tabular}{|c|c|c|c|c|}
\hline & \multicolumn{4}{|c|}{ BMI kg. ${ }^{-2}$} \\
\hline & $<18.5$ & $18.5-<24.0$ & $24.0-<28.0$ & $\geqslant 28.0$ \\
\hline Total $\mathbf{n}$ & \multicolumn{4}{|c|}{452259} \\
\hline Cases $n$ & 1173 & 5809 & 2859 & 898 \\
\hline Rate per 1000 person-years & 7.08 & 2.49 & 1.89 & 1.90 \\
\hline Model 1 & $1.86(1.75-1.99)$ & 1.00 & $0.95(0.91-1.00)$ & $1.12(1.04-1.20)$ \\
\hline Model 2 & $1.75(1.64-1.86)$ & 1.00 & $0.97(0.93-1.02)$ & $1.10(1.02-1.19)$ \\
\hline Model 3 & $1.78(1.66-1.89)$ & 1.00 & $0.89(0.84-0.95)$ & $0.95(0.87-1.05)$ \\
\hline Males n & \multicolumn{4}{|c|}{182209} \\
\hline Cases $\mathrm{n}$ & 651 & 3099 & 1208 & 255 \\
\hline Rate per 1000 person-years & 10.46 & 3.26 & 2.01 & 1.56 \\
\hline Model 1 & $2.05(1.88-2.23)$ & 1.00 & $0.86(0.80-0.92)$ & $0.85(0.75-0.97)$ \\
\hline Model 2 & $1.90(1.74-2.07)$ & 1.00 & $0.89(0.83-0.95)$ & $0.88(0.77-1.00)$ \\
\hline Model 3 & $1.91(1.75-2.08)$ & 1.00 & $0.84(0.77-0.90)$ & $0.76(0.65-0.90)$ \\
\hline Female $n$ & \multicolumn{4}{|c|}{270050} \\
\hline Cases n & 522 & 2710 & 1651 & 643 \\
\hline Rate per 1000 person-years & 5.05 & 1.96 & 1.82 & 2.08 \\
\hline Model 1 & $1.67(1.52-1.84)$ & 1.00 & $1.02(0.96-1.09)$ & $1.27(1.16-1.39)$ \\
\hline Model 2 & 1.59 (1.44-1.75) & 1.00 & $1.02(0.96-1.09)$ & $1.23(1.12-1.34)$ \\
\hline Model 3 & $1.64(1.49-1.81)$ & 1.00 & $0.92(0.85-1.00)$ & $1.05(0.93-1.18)$ \\
\hline
\end{tabular}

Data are presented as HR (95\% CI), unless otherwise stated. Models were adjusted as follows. Model 1: sex (only in total population); model 2: additionally included education (none or primary school, middle or high school, college or university), marital status (married, widowed, divorced or separated, never married), smoking (never or occasional, former and having quit $<5$ years or $\geqslant 5$ years, current smoking $<15$ years, $15-24$ years or $\geqslant 25$ cigarettes $\cdot$ day $^{-1}$ ), passive smoking (never lived with smoker, lived with smoker for $<20$ years, lived with smoker for $\geqslant 20$ years and exposure $<20 \mathrm{~h} \cdot$ week $^{-1}$. lived with smoker for $\geqslant 20$ years and exposure $\geqslant 20 \mathrm{~h} \cdot$ wee $^{-1}$ ), cooking and heating fuel type (coal, wood/charcoal, cleaner fuel, other fuels, no cooking/heating), alcohol drinking (non-drinker, occasional drinker, former drinker, regular drinker), intake frequencies of red meat, fresh fruit and vegetables (daily, 4-6 days.week ${ }^{-1}, 1-3$ days.week ${ }^{-1}$, monthly, rarely or never), physical activity (MET-h.day ${ }^{-1}$ ) and respiratory symptoms (presence or absence); model 3: additionally adjusted for waist circumference. $\mathrm{p}$-value for interaction between sex and $\mathrm{BMI}<0.001$.

circumference and COPD showed a U-shaped relationship, with the lowest risk at waist circumference $\sim 72-75 \mathrm{~cm}$ (figure 1). The association between waist circumference and COPD risk was modified by baseline age, area and level of physical activity (supplementary table S3). Consistent results were observed when using waist-to-hip ratio and waist-to-height ratio as measures of abdominal adiposity (supplementary table S4).

\section{Joint analysis of general and abdominal adiposity}

No statistically significant interaction was found between waist circumference and BMI categories on COPD risk ( $\mathrm{p}$-value for interaction 0.703). Within each BMI category, the risk of COPD increased with waist circumference. Overweight participants with low waist circumference $(<85 \mathrm{~cm}$ for males; $<80 \mathrm{~cm}$ for females) had the lowest risk of COPD (HR 0.87, 95\% CI 0.80-0.95) (table 4). Waist-to-height ratio was also positively associated with COPD, and no significant heterogeneity between BMI categories was found ( $p$-value for interaction 0.462). The association of waist-to-hip ratio with COPD was not consistent across BMI categories ( $p$-value for interaction 0.017), with a negative trend observed in the underweight group.

\section{Sensitivity analysis}

In the sensitivity analyses, the major results remained largely unchanged with excluding participants who developed COPD in the first 3 years of follow-up $(n=1849)$, excluding participants with diabetes at baseline ( $\mathrm{n}=28214)$, or using LLN definition for airflow obstruction. Among never-smokers $(\mathrm{n}=284259)$, the HR (95\% CI) of underweight group was attenuated to 1.64 (1.48-1.81). The risk estimates for abdominal adiposity measures were not substantially altered when restricting the analysis to never-smokers (supplementary table S5).

\section{Discussion}

In this population-based cohort study with 10 years of follow-up, abdominal adiposity defined by waist circumference was associated with a higher risk of COPD hospitalisation or death among Chinese adults. 

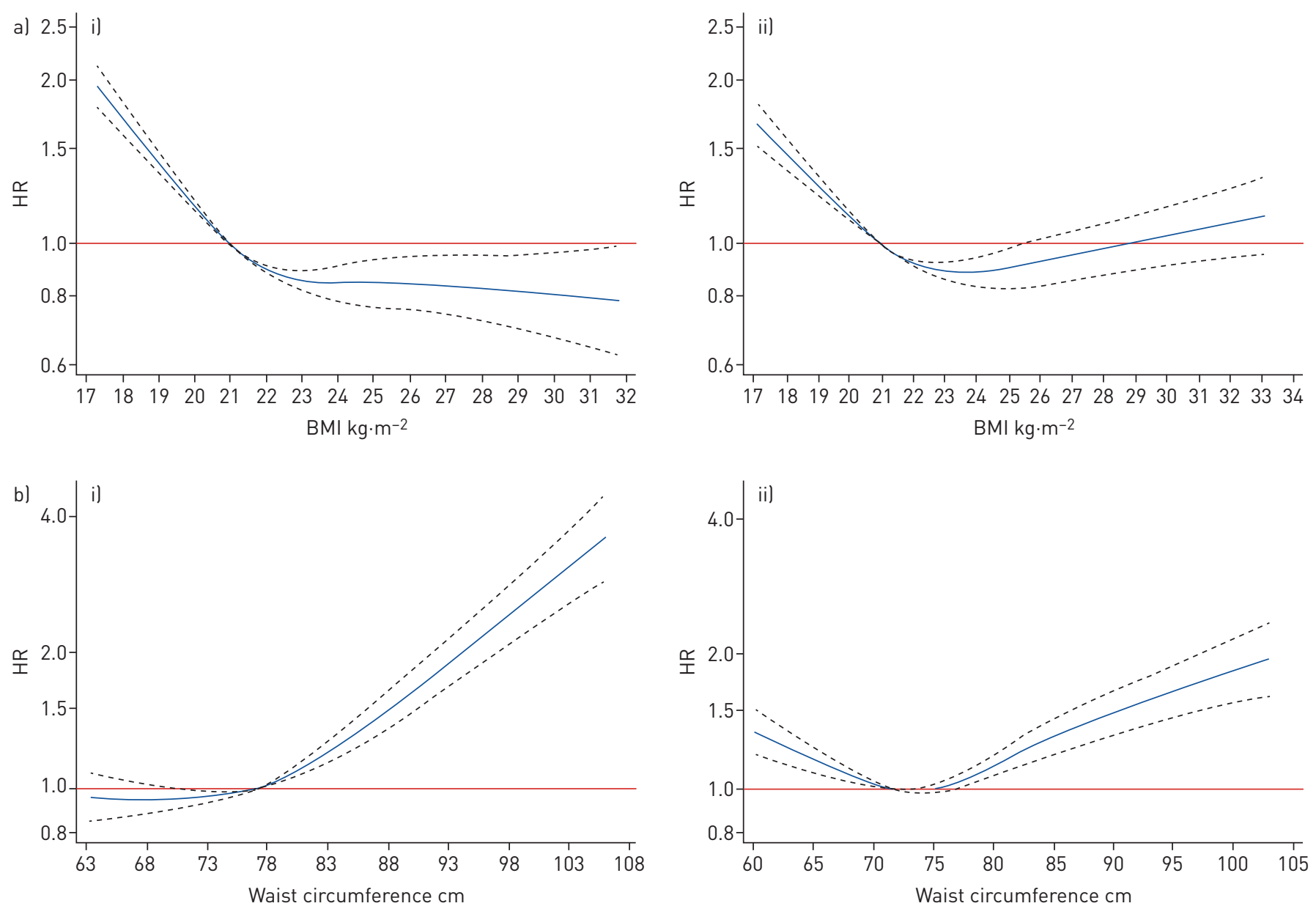

FIGURE 1 Association of a) body mass index (BMI) and b) waist circumference with COPD risk for i) males and ii) females. BMI or waist circumference was included as restricted cubic spline terms in the Cox model, using four knots at the 5th, 35th, 65th and 95th percentiles. Covariate adjustment was based on model 3 . The reference BMI was $21 \mathrm{~kg} \cdot \mathrm{m}^{-2}$. The reference waist circumference was $77 \mathrm{~cm}$ for males and $72 \mathrm{~cm}$ for females. p-values for nonlinearity were all $<0.0001$.

The association was independent of general adiposity defined by BMI. In addition, waist-to-hip ratio and waist-to-height ratio were positively related to the risk of COPD. Low BMI $\left(<18.5 \mathrm{~kg} \cdot \mathrm{m}^{-2}\right)$ appeared to be a significant risk factor for COPD, while overweight (BMI $24.0-<28.0 \mathrm{~kg} \cdot \mathrm{m}^{-2}$ ) and obesity (BMI $\geqslant 28.0 \mathrm{~kg} \cdot \mathrm{m}^{-2}$ ) did not appear to increase COPD risk after adjustment for waist circumference. In males, being overweight or obese was associated with a lower risk of COPD.

Compared with general adiposity, abdominal adiposity (which reflects fat distribution) may be more relevant to COPD risk. Waist circumference is a widely used measurement of abdominal adiposity in epidemiological studies. Many studies have found that high waist circumference was associated with reduced lung function [16-19]. In the National Institutes of Health-American Association of Retired Persons (NIH-AARP) Diet and Health Study, waist circumference showed a positive association with the risk of COPD after adjustment for BMI [6]. Similar results were observed in our Chinese population. Additionally, we evaluated the joint effect of BMI and waist circumference on risk of COPD hospitalisation or death and found no statistically significant interaction. High waist circumference was consistently associated with a higher risk across BMI categories. Overweight people with lower waist circumference were at the lowest risk of COPD, as they had higher muscular mass and lower abdominal fat mass. Furthermore, other measures of abdominal adiposity, including waist-to-hip ratio and waist-to-height ratio were found to be positively associated with COPD in the present study. The possible mechanism underlying the association between abdominal adiposity and COPD is related to inflammation. It has been well known that inflammation plays a crucial role in COPD. As an active endocrine organ, adipose tissue is a source of pro-inflammatory mediators. The increased inflammatory mediators may attract inflammatory cells and amplify the inflammatory process, resulting in structural changes of small airways [20]. Among never-smokers, COPD risk increased with higher waist circumference in our study, 
TABLE 3 Association between baseline waist circumference and the risk of COPD

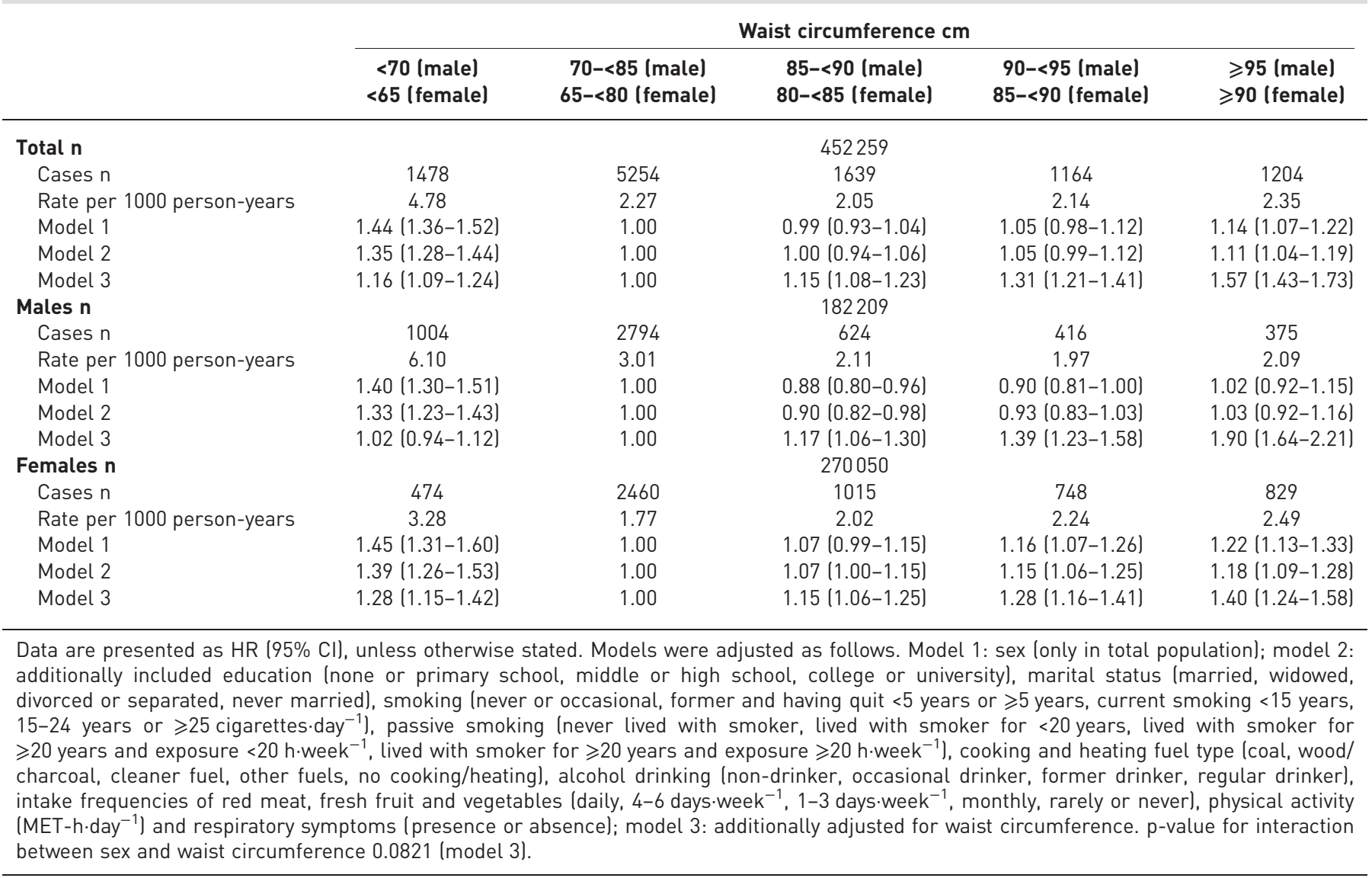

suggesting that abdominal adiposity may be an important risk factor in never-smokers. Reducing abdominal adiposity should be recommended to prevent COPD regardless of smoking status.

Previous literature consistently showed that participants with lower BMI had lower lung function and a higher risk of COPD [21-23]. In line with previous data, our prospective study found that underweight individuals had an increased risk of COPD. In sensitivity analyses, we excluded incident cases in the first 3 years of follow-up to address reverse causation. We also restricted the analysis to never-smokers, as smoking may be a confounder between lower BMI and a higher risk of COPD [24]. Possible explanations include malnutrition, weak resistance against respiratory infection and low muscularity in underweight people [25-27]. The increased risk of COPD among underweight people may be related to sarcopenia, which is frequently observed in COPD patients [28].

Existing study results for the association of general adiposity with COPD are somewhat inconsistent. A meta-analysis found that measures of lung function decreased among obese adults [29]. However, high BMI did not appear to increase COPD risk [4, 21]. In a recent large cross-sectional study in China [2], compared with healthy weight (BMI $18.5-24.9 \mathrm{~kg} \cdot \mathrm{m}^{-2}$ ), overweight and obesity (BMI $\geqslant 25 \mathrm{~kg} \cdot \mathrm{m}^{-2}$ ) was associated with a $25 \%$ decreased risk of COPD prevalence (OR 0.75, 95\% CI 0.62-0.92). In our analysis, being overweight (BMI $24.0-<28.0$ ) or obese $\left(B M I \geqslant 28.0 \mathrm{~kg} \cdot \mathrm{m}^{-2}\right.$ ) was also shown to be a protective factor in males after adjustment for waist circumference. To interpret this result correctly, it is important to understand the meaning of our statistical model. When holding abdominal adiposity constant, BMI primarily reflects lean body mass instead of fatness [30]. Skeletal muscle, a major part of lean body tissues, is positively associated with lung function [27]. Therefore, the lower risk in the overweight group is probably due to the protective effect of muscularity. Similarly, previous studies showed that overweight and obese patients with COPD have a lower mortality rate, known as the "obesity paradox" [31]. Muscular mass could be taken into account to further elucidate the mechanism in the future. We also observed that being overweight was associated with a significantly lower risk of COPD only among those with higher levels of physical activity, indicating that such protective effect could be related to physical activity. Exercising and increasing muscle mass may help prevent COPD. 


\begin{tabular}{|c|c|c|c|c|c|}
\hline & \multicolumn{4}{|c|}{ BMI $\mathrm{kg} \cdot \mathrm{m}^{-2}$} & \multirow{2}{*}{$\begin{array}{l}\mathrm{p} \text {-value fo } \\
\text { interactior }\end{array}$} \\
\hline & $<18.5$ & $18.5-<24.0$ & $24.0-<28.0$ & $\geqslant 28.0$ & \\
\hline \multicolumn{5}{|l|}{ Waist circumference $\mathrm{cm}$} & 0.703 \\
\hline $\begin{array}{l}<85 \text { (male) } \\
<80 \text { (female) }\end{array}$ & $1.77(1.66-1.89)$ & 1.00 & $0.87(0.80-0.95)$ & $1.04(0.59-1.83)$ & \\
\hline $\begin{array}{l}85-<90 \text { (male) } \\
80-<85 \text { (female) }\end{array}$ & $1.91(0.85-4.25)$ & $1.08(1.00-1.17)$ & $0.95(0.88-1.02)$ & $1.18(0.92-1.50)$ & \\
\hline $\begin{array}{l}\geqslant 90 \text { (male) } \\
\geqslant 85 \text { (female) }\end{array}$ & $2.14(0.53-8.57)$ & $1.09(0.95-1.26)$ & $1.08(1.01-1.15)$ & $1.12(1.04-1.21)$ & \\
\hline Waist-to-hip ratio & & & & & 0.017 \\
\hline $\begin{array}{l}<0.9 \text { (male) } \\
<0.8 \text { (female) }\end{array}$ & $1.87(1.72-2.02)$ & 1.00 & $0.81(0.70-0.94)$ & $0.78(0.46-1.32)$ & \\
\hline $\begin{array}{l}0.9-<0.95 \text { (male) } \\
0.8-<0.85 \text { (female) }\end{array}$ & $1.75(1.51-2.01)$ & $1.01(0.94-1.08)$ & $0.90(0.82-0.99)$ & $1.01(0.81-1.26)$ & \\
\hline $\begin{array}{l}\geqslant 0.95 \text { (male) } \\
\geqslant 0.85 \text { (female) }\end{array}$ & $1.58(1.30-1.93)$ & $1.09(1.02-1.17)$ & $1.06(0.99-1.13)$ & $1.17(1.07-1.28)$ & \\
\hline Waist-to-height ratio & & & & & 0.462 \\
\hline$<0.5$ & $1.79(1.67-1.92)$ & 1.00 & $0.87(0.76-0.99)$ & $0.40(0.06-2.84)$ & \\
\hline$\geqslant 0.5$ & $1.78(1.18-2.69)$ & $1.07(1.01-1.13)$ & $1.01(0.96-1.06)$ & $1.13(1.05-1.22)$ & \\
\hline
\end{tabular}

Data are presented as hazard ratio $(95 \%$ CI), unless otherwise stated. Hazard ratios were estimated by joint categorisation of BMI and each measure of abdominal adiposity (waist circumference, waist-to-hip ratio and waist-to-height ratio). Covariate adjustment was based on model 2. p-values for interaction were calculated by likelihood ratio test comparing models with and without a product term of $\mathrm{BMI}$ and abdominal adiposity measures.

To the best of our knowledge, this is the first large cohort study to investigate the association of both general and abdominal adiposity with risk of COPD hospitalisation or death in China. Various anthropometric indexes were objectively measured in the baseline study, allowing us to evaluate the independent effect of general and abdominal adiposity on COPD. Our main strengths include the prospective design, large sample size and stringent quality-control process. We made efforts to reduce bias from reverse causation and confounding by smoking in data analysis. The results of sensitivity analyses indicated that our major findings were reliable.

There are limitations to the present study. The first is the outcome misclassification. Spirometry was not performed on all participants during follow-up, and COPD cases were identified through a health insurance database and death certificates. COPD is often underdiagnosed in China [32], and the underdiagnosis may be more severe among obese individuals [33]. Such misclassification would result in an underestimate of relative risk in obese individuals. Another concern is the low utility of spirometry among COPD cases (13.9\%) [12]. Similarly, only $12.0 \%$ of people with COPD reported a previous pulmonary function test in a recent nationally representative survey [2]. However, most COPD diagnoses (85\%) in our study were supported by different sources of evidence including respiratory symptoms, risk factors and radiological examinations [12]. Second, despite excluding participants with specific pre-existing diseases or those who developed COPD in the first 3 years of follow-up, reverse causation could still not be entirely eliminated due to subclinical conditions. Third, many covariates were self-reported, which may have measurement error. However, the measurements of important confounders such as smoking showed good quality in reproducibility.

In summary, abdominal adiposity is a predictor of COPD risk independent of general adiposity. For people with any level of BMI, maintaining a recommended waist circumference would help prevent COPD if the association is confirmed to be causal. Low BMI $\left(<18.5 \mathrm{~kg} \cdot \mathrm{m}^{-2}\right)$ is also a significant risk factor for COPD. Therefore, maintaining a healthy BMI (between $18.5-24 \mathrm{~kg} \cdot \mathrm{m}^{-2}$ ) and avoiding abdominal adiposity should be both considered in the management of body weight to reduce the future risk of COPD. Our data provide useful information for determining optimal BMI and waist circumference cut-off points among Chinese people.

Acknowledgements: The most important acknowledgement is to the participants in the study and the members of the survey teams in each of the 10 regional centres, as well as to the project development and management teams based at Beijing, Oxford and the 10 regional centres. 
Conflict of interest: None declared.

Support statement: This work was supported by grants (2016YFC1303904, 2016YFC0900500, 2016YFC0900501, 2016YFC0900504) from the National Key R\&D Program of China. The CKB baseline survey and the first re-survey were supported by a grant from the Kadoorie Charitable Foundation in Hong Kong. The long-term follow-up is supported by grants from the UK Wellcome Trust (212946/Z/18/Z, 202922/Z/16/Z, 104085/Z/14/Z, 088158/Z/09/Z), National Natural Science Foundation of China $(91846303,91843302,81390540,81390541,81390544)$, and Chinese Ministry of Science and Technology (2011BAI09B01). The funders had no role in the study design, data collection, data analysis and interpretation, writing of the report, or the decision to submit the article for publication. Funding information for this article has been deposited with the Crossref Funder Registry.

\section{References}

1 GBD 2016 Causes of Death Collaborators. Global, regional, and national age-sex specific mortality for 264 causes of death, 1980-2016: a systematic analysis for the Global Burden of Disease Study 2016. Lancet 2017; 390: $1151-1210$.

2 Wang C, Xu J, Yang L, et al. Prevalence and risk factors of chronic obstructive pulmonary disease in China (the China Pulmonary Health [CPH] study): a national cross-sectional study. Lancet 2018; 391: 1706-1717.

3 Jensen MK, Chiuve SE, Rimm EB, et al. Obesity, behavioral lifestyle factors, and risk of acute coronary events. Circulation 2008; 117: 3062-3069.

4 Smith M, Li L, Augustyn M, et al. Prevalence and correlates of airflow obstruction in $~ 317,000$ never-smokers in China. Eur Respir J 2014; 44: 66-77.

5 Guerra S, Sherrill DL, Bobadilla A, et al. The relation of body mass index to asthma, chronic bronchitis, and emphysema. Chest 2002; 122: 1256-1263.

6 Behrens G, Matthews CE, Moore SC, et al. Body size and physical activity in relation to incidence of chronic obstructive pulmonary disease. CMAJ 2014; 186: E457-E469.

7 Deurenberg P, Deurenberg-Yap M, Guricci S. Asians are different from Caucasians and from each other in their body mass index/body fat per cent relationship. Obes Rev 2002; 3: 141-146.

8 Chen Z, Lee L, Chen J, et al. Cohort profile: The Kadoorie Study of Chronic Disease in China (KSCDC). Int J Epidemiol 2005; 34: 1243-1249.

9 Chen Z, Chen J, Collins R, et al. China Kadoorie Biobank of 0.5 million people: survey methods, baseline characteristics and long-term follow-up. Int J Epidemiol 2011; 40: 1652-1666.

10 Chen Z, Smith M, Du H, et al. Blood pressure in relation to general and central adiposity among 500000 adult Chinese men and women. Int J Epidemiol 2015; 44: 1305-1319.

11 Standardization of Spirometry, 1994 Update. American Thoracic Society. Am J Respir Crit Care Med 1995; 152: $1107-1136$.

12 Kurmi OP, Vaucher J, Xiao D, et al. Validity of COPD diagnoses reported through nationwide health insurance systems in the People's Republic of China. Int J Chron Obstruct Pulmon Dis 2016; 11: 419-430.

13 Zhou BF, Cooperative Meta-Analysis Group of the Working Group on Obesity in China. Predictive values of body mass index and waist circumference for risk factors of certain related diseases in Chinese Adults - study on optimal cut-off points of body mass index and waist circumference in Chinese adults. Biomed Environ Sci 2002; 15: 83-96.

14 Chen C, Lu D, Department of Disease Control, Ministry of Health, PR China. The guidelines for prevention and control of overweight and obesity in Chinese adults. Biomed Environ Sci 2004; 17: 1-36.

15 World Health Organization. Waist Circumference and Waist-Hip Ratio: Report of a WHO Expert Consultation. Geneva, WHO Press, 2011.

16 Ochs-Balcom HM, Grant BJB, Muti P, et al. Pulmonary function and abdominal adiposity in the general population. Chest 2006; 129: 853-862.

17 Chen Y, Rennie D, Cormier YF, et al. Waist circumference is associated with pulmonary function in normal-weight, overweight, and obese subjects. Am J Clin Nutr 2007; 85: 35-39.

18 Vatrella A, Calabrese C, Mattiello A, et al. Abdominal adiposity is an early marker of pulmonary function impairment: findings from a Mediterranean Italian female cohort. Nutr Metab Cardiovasc Dis 2016; 26: 643-648.

19 Wannamethee SG, Shaper AG, Whincup PH. Body fat distribution, body composition, and respiratory function in elderly men. Am J Clin Nutr 2005; 82: 996-1003.

20 Barnes PJ. Cellular and molecular mechanisms of chronic obstructive pulmonary disease. Clin Chest Med 2014; 35: 71-86.

21 Lee SJ, Kim SW, Kong KA, et al. Risk factors for chronic obstructive pulmonary disease among never-smokers in Korea. Int J Chron Obstruct Pulmon Dis 2015; 10: 497-506.

22 Zhou Y, Wang D, Liu S, et al. The association between BMI and COPD: the results of two population-based studies in Guangzhou, China. COPD 2013; 10: 567-572.

23 Harik-Khan RI, Fleg JL, Wise RA. Body mass index and the risk of COPD. Chest 2002; 121: 370-376.

24 Albanes D, Jones DY, Micozzi MS, et al. Associations between smoking and body weight in the US population: analysis of NHANES II. Am J Public Health 1987; 77: 439-444.

25 Shaheen S, Barker DJ. Early lung growth and chronic airflow obstruction. Thorax 1994; 49: 533-536.

26 Dobner J, Kaser S. Body mass index and the risk of infection - from underweight to obesity. Clin Microbiol Infect 2018; 24: 24-28.

27 Park C-H, Yi Y, Do JG, et al. Relationship between skeletal muscle mass and lung function in Korean adults without clinically apparent lung disease. Medicine 2018; 97: e12281.

28 Benz E, Trajanoska K, Lahousse L, et al. Sarcopenia in COPD: a systematic review and meta-analysis. Eur Respir Rev 2019; 28: 190049.

29 Forno E, Han YY, Mullen J, et al. Overweight, obesity and lung function in children and adults - a meta-analysis. J Allergy Clin Immunol Pract 2018; 6: 570-581.

30 Hu FB. Obesity Epidemiology. Oxford, Oxford University Press, 2008. 
31 Hancu A. Nutritional status as a risk factor in COPD. Maedica 2019; 14: 140-143.

32 Zhong N, Wang C, Yao W, et al. Prevalence of chronic obstructive pulmonary disease in China: a large, population-based survey. Am J Respir Crit Care Med 2007; 176: 753-760.

33 Çolak Y, Marott JL, Vestbo J, et al. Overweight and obesity may lead to under-diagnosis of airflow limitation: findings from the Copenhagen City Heart Study. COPD 2015; 12: 5-13. 